\title{
OrfG, a VirB8-like protein encoded by an integrative and conjugative element in S. thermophilus, has a trimeric architecture with intertwined subunits that may be involved in the Gram-positive Conj-T4SS
}

\author{
Julien Cappele ${ }^{1}$, Badreddine Douzi ${ }^{2}$, Sandrine Mathiot ${ }^{1}$, Nathalie Leblond-Bourget ${ }^{2}$, Claude Didierjean ${ }^{1}$, \\ Frédérique Favier ${ }^{1}$ \\ ${ }^{1} C R M^{2}$, Nancy, France; \\ ${ }^{2}$ Université de Lorraine, INRAE, DynAMic, Nancy, France;
}

julien.cappele@univ-lorraine.fr

\begin{abstract}
Antibiotic resistance is usually shared between bacteria by using conjugation [1], where a bacterium transfers part of its genome to another bacterium by involving a complex machinery called a secretion system. Conjugative Type IV secretion systems (Conj-T4SSs) are one of the most prevalent ways to share DNA between prokaryotes. Integrative and conjugative elements (or ICEs) are one of the principal types of mobile genetic elements that enable horizontal transmission of genetic information. ICEs encode their own ConjT4SSs to perform conjugation and have the particularity to be integrated into the chromosome of the host cell. While descriptions of Conj-T4SSs in Gram-negative bacteria are available from CryoEM experiments, no data exist for Gram-positive bacteria yet. In Streptococcus thermophilus, ICESt3 encodes 14 putative proteins involved in the conjugation process. Among them, the Gramnegative VirB8-equivalent protein, called OrfG, is expected to be essential in the plasma membrane part of T4SS since it is supposed to act as an interaction hub for other T4SS proteins. OrfG contains three domains, one transmembrane domain and two soluble ones. Here we present the X-Ray structures of the soluble domains of OrfG. We performed an in-depth analysis of all VirB8-like domains [2] by using existing and new multi-protein structural alignment visualization tools. One of these tools, called MPSA_Viewer, was developed in our lab and significantly improved the visual readability of multi-protein structural alignments, especially when sequence identities are very low. We also analyzed the quaternary structure of OrfG, which consists of a trimeric assembly of interwoven monomers. The trimeric organization seems specific to VirB8-like proteins of Gram-positive bacteria since two other occurrences were found in the structural database. Such intricated assemblies can be biologically relevant, as observed for instance in the prefusion conformation of the 2019-nCoV spike [3]. We also noticed that the variable spacing at the center of these Gram-positive trimers might be compatible with substrate translocation [4] if other conditions are met.
\end{abstract}

[1] Frost, L. S., Leplae, R., Summers, A.O. \& Toussaint, A. (2005) Nat. Rev. Microbiol. 3, 722.

[2] Cappèle, J., Mohamad Ali, A., Leblond-Bourget, N., Mathiot, S., Dhalleine, T., Payot, S., Savko, M. Didierjean, C., Favier, F. \& Douzi, B. (2021). Front. Mol. Biosci., 8, 642606.

[3] Wrapp D, Wang, N. Corbett, K.S., Goldsmith, J.A., Hsieh, C.L., Abiona, O., Graham, B.S. \& McLellan, J.S. (2020). Science, 367, 1260.

[4] Miletic, S., Fahrenkamp, D., Goessweiner-Mohr, N., Wald, J., Pantel, M., Vesper, O., Kotov, V. \& Marlovits, T.C. (2021) Nat. Commun. 12, 1546.

Keywords: x-ray biocrystallography, type IV secretion system, VirB8, multiple protein structural alignements 